\title{
Consensus on the use of DPP4 Inhibitors and SGLT2 Inhibitors in T2DM with Chronic Kidney Disease-from Indian Context
}

Amit Gupta ${ }^{1}$, Gokulnath ${ }^{2 *}$, Vijay Kher ${ }^{3}$, Manisha Sahay ${ }^{4}$, Umesh Khanna ${ }^{5}$,
Alan Almeida ${ }^{6}$, Tarun Jeloka ${ }^{7}$, Manish Rathi ${ }^{8}$, Sanjay Maitra ${ }^{9}$, Arpita Ray
Chaudhury $^{10}$, Narayan Prasad ${ }^{11}$ and Kaushik Mandal ${ }^{12}$
${ }^{1}$ Director and HOD, Department of Nephrology \& Transplant, Apollo Hospital, India
${ }^{2}$ Director of Nephrology Services, Apollo Hospitals, India

${ }^{3}$ Chairman, Division of Nephrology and Kidney Transplant Medicine, Medanta Kidney and

Urology Institute, India

${ }^{4}$ Professor \& Head, Department of Nephrology, Osmania Medical College and General Hospital, India

${ }^{5}$ Director Kidney Associates, Lancelot Kidney \& GI Centre, India

${ }^{6}$ Nephrologist, P. D. Hinduja Hospital \& Research Center, India

${ }^{7}$ Director of Nephrology, Aditya Birla Memorial Hospital, India

${ }^{8}$ Professor, Department of Nephrology, Post Graduate Institute of Medical Education \& Research, India

${ }^{9}$ Senior Nephrologist, Apollo Hospital, India

${ }^{10}$ Professor, Department of Nephrology, Institute of Post Graduate Medical Education \& Research, India

${ }^{11}$ Professor \& HOD, Department of Nephrology, Sanjay Gandhi Post Graduate Institute of Medical Sciences, India

${ }^{12}$ Manager, Medical Affairs, AstraZeneca Pharma India Ltd, India

*Corresponding author: Dr. Gokulnath, Director of Nephrology Services, Apollo Hospitals, Bangalore, India, Email: gokulnath. neph@gmail.com

\section{Abstract}

Chronic Kidney Disease and Diabetes are diseases of concern globally including developing countries like India. The delayed intervention of non-communicable disease management, the unique socioeconomic condition, etc makes chronic kidney disease a potential threat in the future. Diabetes in India plays an important role in developing CKD \& its associated complications. To treat diabetes, many innovative antidiabetic agents were introduced in the last two decades. Some of these newer anti-diabetic medications have established cardio-renal safety in diabetic patients. Many endocrine and cardiovascular societies have already adopted these anti-diabetic agents into the respective algorithms. Overall for newer anti-diabetic medication, there is no available published guidance in India that has prioritized both renal safety and other parameters related to nephrology practice. A group of Indian nephrologists, after analyzing existing published evidence and guidance from different guidelines, prepared a simple common algorithm to manage diabetes patients with associated CKD. This new algorithm, having both DPP4i and SGLT2i, will surely help Indian health care professionals to manage diabetes in CKD patients, in a more efficient way.

Keywords: Diabetes; Chronic Kidney Disease; Diabetes Mellitus; Cardiovascular 


\section{Diabetes \& Obesity International Journal}

Abbreviations: CREDENCE Trial: Canagliflozin and Renal Events in Diabetes with Established Nephropathy Clinical Evaluation; DECLARE-TIMI 58: Dapagliflozin Effect on Cardiovascular Events-Thrombolysis in Myocardial Infarction 58; CANVAS Programme: CANagliflozin cardioVascular Assessment Study (CANVAS) Program; CARMELINA Trial: Cardiovascular and Renal Microvascular Outcome Study With Linagliptin in Patients with Type 2 Diabetes Mellitus; DAPA-HF: Dapagliflozin on the Incidence of Worsening Heart Failure or Cardiovascular Death in Patients With Chronic Heart Failure; DAPA-CKD: Dapagliflozin on Renal Outcomes and Cardiovascular Mortality in Patients With Chronic Kidney Disease; EMPAREG Trial: Empagliflozin Cardiovascular Outcome Event Trial in Type 2 Diabetes Mellitus Patients-Removing Excess Glucose; EMPEROR-Reduced: Empagliflozin Outcome Trial in Patients with Chronic Heart Failure with Reduced Ejection Fraction; MARLINA-T2D: Efficacy, Safety \& Modification of Albuminuria in Type 2 Diabetes Subjects With Renal Disease With LINAgliptin; SAVOR-TIMI 53: Saxagliptin and cardiovascular outcomes in patients with type 2 diabetes mellitus; TECOS: Trial Evaluating Cardiovascular Outcomes with Sitagliptin; AACE: American Association of Clinical Endocrinology; ACE:Angiotensin Converting Enzyme; ADA: American Diabetic Association; AKI: Acute Kidney Injury; ARB:Angiotensin Receptor Blocker; ASCVD:Atherosclerotic Cardiovascular Disease; CCF: Congestive Cardiac Failure; CI: Confidence Interval; CKD : Chronic Kidney Disease; CV-Cardiovascular; CVOT: Cardiovascular Outcome Trial; DKA: Diabetic Ketoacidosis; DPP4i: Dipeptidyl peptidase-4 inhibitor; eGFR: Estimated Glomerular Filtration Rate; EMA: European Medicines Agency.

\section{Introduction}

Chronic Kidney Disease \& diabetes are two of the important \& independent causes of increased mortality and morbidity globally. Due to the alarming enhancement of burden in the last few decades, these two diseases are in the focus of public health priority worldwide [1]. The burden of both these diseases is very high in developing countries like India, with a large number of patients being below the poverty line. The lower allocation of gross domestic product to the healthcare sector, inadequate \& improper distribution of health care facilities, less availability of skilled health care personnel, etc all contribute to sub-optimal outcomes while managing these chronic diseases in India. The challenges of managing both these diseases in India is quite different from the rest of the world $[2,3]$.

In the last decade, different agents of these newer anti- diabetic agents- DPP4i and SGLT2i established cardiovascular \& renal safety in landmark trials. Their use has been endorsed by different endocrine and cardiological society globally. But, there is no published guideline combining both SGLT2 $\mathrm{i}$ and DPP4i, based on prioritized parameters related to nephology segment (e.g. renal protection evidence, infection, and other factors related to nephrology). So, there is a felt need for consensus document/guideline for Indian CKD patients with T2DM updating guidance to use these two innovative class of anti-diabetic agents.

\section{Methodology}

Clinical trials and review articles published in Pub Med, Embase, Google Scholar, and other indexed and peerreviewed journals were reviewed to identify publications and other key data. The studies related to the subject of interest were searched in the search section with terms 'CKD DPP4i/ SGLT2i/ Renal safety. Clinical trials and review articles published in an indexed and peer-reviewed journal till $30^{\text {th }}$ Sep 2020, were reviewed by the Expert Panel to identify publications and other key data that would help in guideline recommendations. Expert Panel members also critically evaluated the currently available recommendations available in the public domain. The consensus statement and recommendation of the nephrology group were arrived at by deliberations based on this evidence.

\section{Evidence of DPP4 Inhibitors in Renal Protection}

Many studies have demonstrated a reduction in albuminuria with DPP4i. Only some of the DPP4i have shown renal protection (viz. decrease in Doubling of Sr. Creatinine, $>50 \%$ reduction of eGFR, the number of patients initiating on long term dialysis, etc). Three important randomized clinical trials assessed the protective effect of DPP-4 inhibitors on renal functions. The first published study, SAVOR-TIMI 53, the largest CVOT with DPP4i, had patients across CKD stages (except dialysis patient at baseline). In this trial, UACR reduction was significantly better in Saxagliptin arm compared to placebo, irrespective of glycaemic improvement. The renal safety endpoints (Composite of doubling of sr. creatinine, initiation of dialysis or transplant, sr. creatinine $>6$ $\mathrm{mg} / \mathrm{dl}$, death) were analysed (post hoc analysis). The trial established the renal safety of Saxagliptin in diabetic CKD patients [4-6]. Hospitalization of heart failure event was inconsistent with saxagliptin in different eGFR categories ( $<30 \mathrm{ml} / \mathrm{min}, 30-50 \mathrm{ml} / \mathrm{min} \mathrm{\&}>50 \mathrm{ml} / \mathrm{min}$ ). Especially in low eGFR $(<30 \mathrm{ml} / \mathrm{min})$ with high-risk category, HHF was similar to placebo [7] (Table 1, Table 2 and Figure 1)). 
Diabetes \& Obesity International Journal

\begin{tabular}{|c|c|c|c|c|c|}
\hline & Total N (\%) & Worsened N (\%) & No Change $\mathrm{N}(\%)$ & Improved N (\%) & p-value* \\
\hline \multicolumn{5}{|c|}{ eGFR $>50 \mathrm{~mL} / \mathrm{min} / 1.73 \mathrm{~m}^{2}(\mathrm{n}=10,621)$} & \multirow{3}{*}{$<0.0001$} \\
\hline Saxagliptin & $5,380(50.7 \%)$ & $682(12.7 \%)$ & $4,139(76.9 \%)$ & $559(10.4 \%)$ & \\
\hline Placebo & $5,241(49.3 \%)$ & $790(15.1 \%)$ & $4,003(76.4 \%)$ & $448(8.5 \%)$ & \\
\hline \multicolumn{2}{|c|}{ eGFR $\leq 50 \mathrm{~mL} / \mathrm{min} / 1.73 \mathrm{~m}^{2}(\mathrm{n}=1739)$} & & & & \multirow{3}{*}{0.041} \\
\hline Saxagliptin & $885(50.9 \%)$ & $151(17.1 \%)$ & $623(70.4 \%)$ & $111(12.5 \%)$ & \\
\hline Placebo & $854(49.1 \%)$ & $179(21.0 \%)$ & $591(69.2 \%)$ & $84(9.8 \%)$ & \\
\hline
\end{tabular}

Table 1: Frequency of progressive microalbuminuria by the completion of follow-up according to renal function (SAVOR-TIMI 53).

Note: The risk of progressive microalbuminuria was defined as a treatment difference in the number and proportion of patients with worsening, no change, or improvement in the urinary albumin-to-creatinine ratio (ACR), defined as a shift from baseline category (33.9 mg/mmol) throughout follow-up among patients with complete data. *P-values based on chisquare or Fisher's exact test.

\begin{tabular}{|c|c|c|c|c|c|c|c|}
\hline \multirow[t]{2}{*}{ Endpoint } & \multicolumn{3}{|c|}{$\mathrm{eGFR}>50 \mathrm{~mL} / \mathrm{min} / 1.73 \mathrm{~m}^{2}$} & \multicolumn{3}{|c|}{$\mathrm{eGFR} \leq 50 \mathrm{~mL} / \mathrm{min} / 1.73 \mathrm{~m}^{2}$} & \multirow[b]{2}{*}{ (Interaction) } \\
\hline & $\begin{array}{c}\text { Saxagliptin } \\
5 \mathrm{mg} \\
(\mathrm{n}=6986)\end{array}$ & $\begin{array}{c}\text { Placebo } \\
(\mathrm{n}= \\
6930)\end{array}$ & $\begin{array}{l}\text { Hazard } \\
\text { Ratio } \\
(95 \% \mathrm{CI})\end{array}$ & $\begin{array}{c}\text { Saxaglipti } \\
\mathrm{n} \\
2.5 \mathrm{mg} \\
(\mathrm{n}=1294)\end{array}$ & $\begin{array}{c}\text { Placebo }(\mathrm{n}= \\
1282)\end{array}$ & $\begin{array}{l}\text { Hazard Ratio } \\
\quad(95 \% \mathrm{CI})\end{array}$ & \\
\hline $\begin{array}{c}\text { Renal Composite } \\
\text { Endpoints (Doubling of } \\
\text { Serum Creatinine, } \\
\text { Dialysis, Renal } \\
\text { Transplant, Serum } \\
\text { Creatinine }>6 \mathrm{mg} / \mathrm{dL} \text { ) }\end{array}$ & $1.50 \%$ & $1.22 \%$ & $\begin{array}{c}1.10(0.83- \\
1.44)\end{array}$ & $5.80 \%$ & $6.16 \%$ & $\begin{array}{l}1.06(0.78- \\
1.44)\end{array}$ & 0.90 \\
\hline $\begin{array}{l}\text { Renal Composite End } \\
\text { points + Death }\end{array}$ & $5.24 \%$ & $4.41 \%$ & $\begin{array}{c}1.10(0.95- \\
1.27)\end{array}$ & $13.90 \%$ & $13.70 \%$ & $\begin{array}{l}1.06(0.86- \\
1.29)\end{array}$ & 0.78 \\
\hline
\end{tabular}

Table 2: Renal safety endpoint analysis in SAVOR-TIMI trial.

Note: Abbreviations: CI indicates- confidence interval; HR- hazard ratio; Hosp- hospitalization; eGFRestimated glomerular filtration rate. Interaction P-values based on categorical eGFR groups.

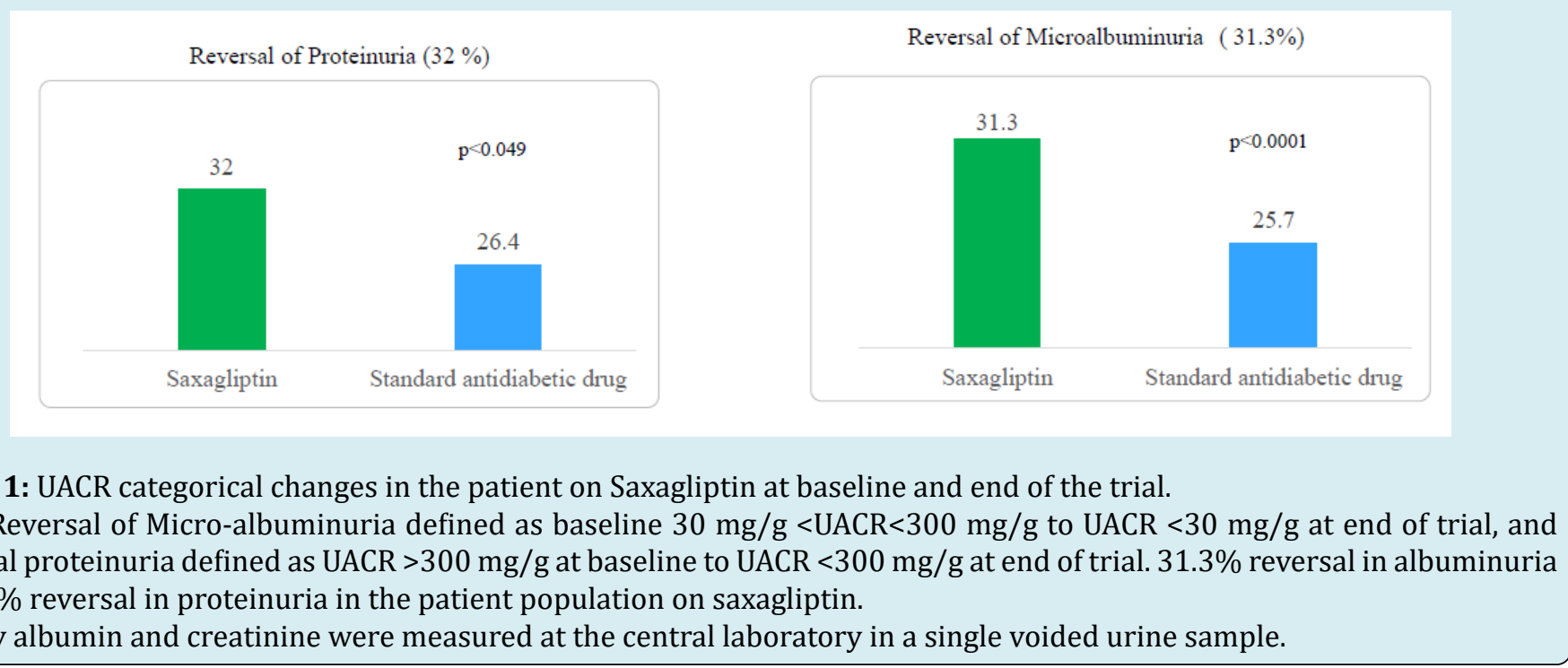


Linagliptin established cardiovascular safety in CARMELINA Trial in the patient population of type 2 diabetes with or without CKD patient up to CKD stage 4 (eGFR $>15 \mathrm{ml} / \mathrm{min}$ ). Apart from the MARLINA-T2D trial, linagliptin also established significant improvement in UACR in CARMELINA trial compared to placebo. CARMELINA trial also established renal safety based on predefined renal safety as a secondary endpoint [8-10] (Table 3, Figures 2 \& 3). Important cardiovascular outcome trials with DPP4i have been summarised in supplementary Table 4. Saxagliptin and Linagliptin are the only two DPP4i with established renal safety (by Renal composite endpoints- Doubling of Serum Creatinine, Dialysis, Renal Transplant, etc).

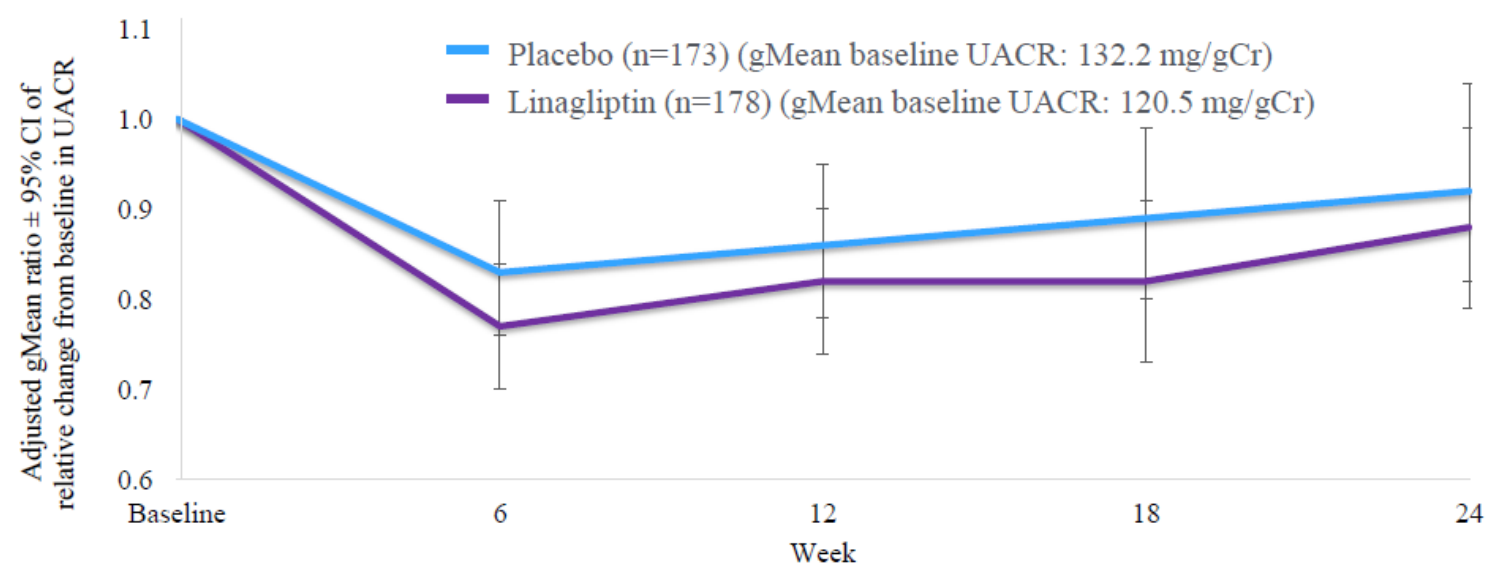

Figure 2: Change in UACR from baseline over time in MARLINA-T2D Trial.

Note: (Figure 2) The adjusted geometric mean ratio of relative change from baseline in UACR over time, FAS- (LOCF) [ FAS=Full Analysis Set, LOCF= Last Observation Carried Forward].

No significant difference between linagliptin and placebo in the change in UACR from baseline over time.

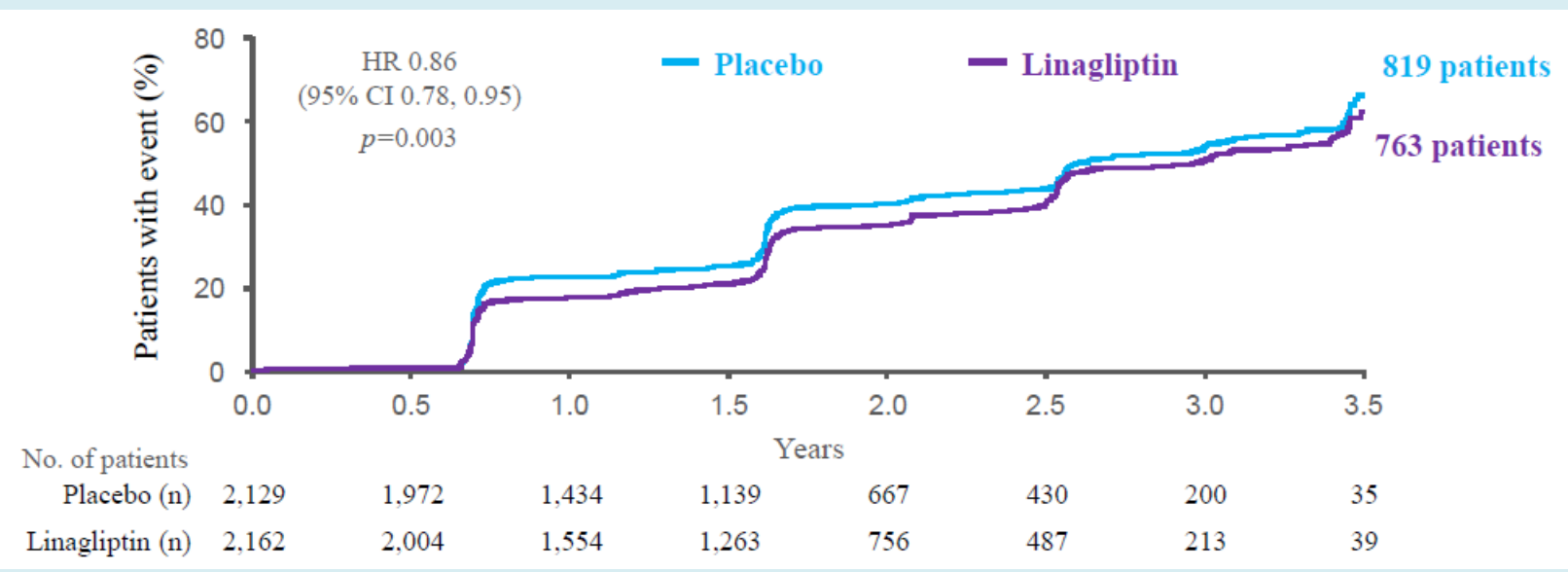

Figure 3: Time to the first occurrence of albuminuria progression* (CARMELINA Trial).

Note : Linagliptin event rate 21.36/100 PY Placebo event rate 2.4.54/100 PY.

Treated set, Kaplan-Meier estimate. Hazard ratio and $95 \%$ CI based on the Cox regression model with terms for the treatment group ( $\mathrm{p}=0.0034)$ and region $(\mathrm{p}<0.0001)$

*change from normo- to micro- or macroalbuminuria, or from micro- to macroalbuminuria; 
Diabetes \& Obesity International Journal

\begin{tabular}{|c|c|c|c|c|c|c|c|}
\hline & \multicolumn{2}{|c|}{ Linagliptin $(n=3494)$} & \multicolumn{2}{|c|}{ Placebo $(n=3485)$} & \multirow{2}{*}{$\begin{array}{l}\text { Incidence rate } \\
\text { difference } \\
\text { Linagliptin- } \\
\text { Placebo(95\% } \\
\text { CI) }\end{array}$} & \multirow[b]{2}{*}{$\begin{array}{c}\text { Hazard } \\
\text { Ratio }(95 \% \mathrm{CI})\end{array}$} & \multirow[b]{2}{*}{$\begin{array}{c}\text { p- } \\
\text { value }\end{array}$} \\
\hline & No (\%) & $\begin{array}{c}\text { Rate per } \\
100 \\
\text { patient } \\
\text { years } \\
\end{array}$ & No (\%) & $\begin{array}{c}\text { Rate per } \\
100 \text { patient } \\
\text { years }\end{array}$ & & & \\
\hline $\begin{array}{l}\text { Sustained SRD, death } \\
\text { due to Kidney Failure, or } \\
\text { sustained decrease of } \\
>50 \% \text { in eGFR from } \\
\text { Baseline }\end{array}$ & $230(6.6)$ & 3.39 & $227(6.5)$ & 3.42 & $\begin{array}{c}-0.03(-0.65 \text { to } \\
0.60)\end{array}$ & $0.98(0.82-1.18)$ & 0.87 \\
\hline $\begin{array}{l}\text { Death due to renal failure } \\
\text { or sustained ESRD }\end{array}$ & $136(3.9)$ & 1.78 & $154(4.4)$ & 2.04 & $\begin{array}{c}-0.26(-0.70 \text { to } \\
0.18)\end{array}$ & $0.87(0.69-1.10)$ & 0.24 \\
\hline Albuminuria progression & $763(35.3)$ & 21.36 & $819(38.5)$ & 24.54 & $\begin{array}{c}-3.18(-5.44 \text { to }- \\
0.92)\end{array}$ & $0.86(0.78-0.95)$ & 0.003 \\
\hline $\begin{array}{c}\text { Composite microvascular } \\
\text { end point }\end{array}$ & $785(36.3)$ & 22.14 & $843(39.6)$ & 25.42 & $\begin{array}{c}-3.28(-5.59 \text { to }- \\
0.97)\end{array}$ & $0.86(0.78-0.95)$ & 0.003 \\
\hline
\end{tabular}

Table 3: Kidney and Microvascular Outcomes (Carmelina Trial).

\begin{tabular}{|c|c|c|c|c|}
\hline & SAVOR-TIMI-53(4) & EXAMINE (12) & $\operatorname{TECOS}(13)$ & CARMELINA(11) \\
\hline & $(n=16,492)$ & $(n=5,380)$ & $(n=14,671)$ & $(n=6,979)$ \\
\hline Intervention & Saxagliptin/Placebo & Alogliptin /placebo & Sitagliptin/Placebo & Linagliptin/Placebo \\
\hline Main Inclusion Criteria & $\begin{array}{l}\text { Type } 2 \text { Diabetes and } \\
\text { history of or multiple } \\
\text { risk factors for CVD }\end{array}$ & $\begin{array}{l}\text { Type } 2 \text { diabetes and } \\
\text { ACS within 15-90 days } \\
\text { before randomization }\end{array}$ & $\begin{array}{c}\text { Type } 2 \text { Diabetes } \\
\text { and preexisting } \\
\text { CVD }\end{array}$ & $\begin{array}{c}\text { Type } 2 \text { Diabetes and } \\
\text { high } \\
\mathrm{CV} \text { and renal risk }\end{array}$ \\
\hline A1C Inclusion Criteria (\%) & $>6.5$ & $6.5-11.0$ & $6.5-8.0$ & $6.5-10.0$ \\
\hline Age (Years) ${ }^{* *}$ & 65.1 & 61 & 65.4 & 65.8 \\
\hline Race(\%White) & 75.2 & 72.7 & 67.9 & 80.2 \\
\hline Sex (\%Male) & 66.9 & 57.9 & 70.7 & 62.9 \\
\hline $\begin{array}{c}\text { Diabetes Duration } \\
\text { (Years) }^{* *}\end{array}$ & 10.3 & 7.1 & 11.6 & 14.7 \\
\hline $\begin{array}{l}\text { Median follow-up period } \\
\text { (Years) }\end{array}$ & 2.1 & 1.5 & 3 & 2.2 \\
\hline Statin Use (\%) & 78 & 91 & 80 & 71.8 \\
\hline Metformin Use (\%) & 70 & 66 & 82 & 54.8 \\
\hline Prior CVD/CHF (\%) & $78 / 13$ & $100 / 28$ & $74 / 18$ & $57 / 26.8$ \\
\hline Mean Baseline A1C (\%) & 8 & 8 & 7.2 & 7.9 \\
\hline $\begin{array}{l}\text { Mean difference in } \mathrm{A} 1 \mathrm{C} \\
\text { (Between groups at end of } \\
\text { treatment }(\%) \text { ) }\end{array}$ & -0.3 & -0.3 & -0.3 & -0.36 \\
\hline Year started/reported & $2010 / 2013$ & $2009 / 2013$ & $2008 / 2015$ & $2013 / 2018$ \\
\hline Primary Outcome\$ & $\begin{array}{l}\text { 3- Point MACE } 1.00 \\
(0.89-1.12)\end{array}$ & $\begin{array}{l}\text { 3-Point MACE } 0.96 \\
\text { (95\% UL <1.16) }\end{array}$ & $\begin{array}{c}\text { 4-Point MACE } 0.98 \\
(0.89-1.08)\end{array}$ & $\begin{array}{l}\text { 3-point MACE } 1.02 \\
(0.89-1.17)\end{array}$ \\
\hline
\end{tabular}


Diabetes \& Obesity International Journal

\begin{tabular}{|c|c|c|c|c|}
\hline Key secondary outcome\$ & $\begin{array}{c}\text { Expanded MACE 1.02 } \\
(0.94-1.11)\end{array}$ & $\begin{array}{c}\text { 4-Point MACE 0.95 } \\
(95 \% \text { UL <1.14) }\end{array}$ & $\begin{array}{c}\text { 3-point MACE 0.99 } \\
(0.89-1.12)\end{array}$ & $\begin{array}{c}\text { Kidney composite } \\
\text { (ESRD, Sustained }> \\
40 \% \text { decrease } \\
\text { in eGFR, or renal death) } \\
1.04(0.89-1.22)\end{array}$ \\
\hline Cardiovascular Death\$ & $1.03(0.87-1.22)$ & $0.85(0.66-1.10)$ & $1.03(0.89-1.19)$ & $0.96(0.81-1.14)$ \\
\hline MI\$ & $0.95(080-1.12)$ & $1.08(0.88-1.33)$ & $0.95(0.81-1.11)$ & $1.12(0.09-1.40)$ \\
\hline Stroke\$ & $1.11(1.07-1.51)$ & $0.91(0.55-1.50)$ & $0.97(0.79-1.19)$ & $0.91(0.67-1.23)$ \\
\hline HF Hospitalization\$ & $1.27(1.07-1.51)$ & $1.19(0.90-1.58)$ & $1.00(0.83-1.20)$ & $0.90(0.74-1.08)$ \\
\hline $\begin{array}{c}\text { Unstable Angina } \\
\text { Hospitalization\$ }\end{array}$ & $1.19(0.89-1.60)$ & $0.90(0.60-1.37)$ & $0.90(0.70-1.16)$ & $0.87(0.57-1.31)$ \\
\hline All-cause mortality\$ & $1.11(0.96-1.27)$ & $0.88(0.71-1.09)$ & $1.01(0.90-1.14)$ & $0.98(0.84-1.13)$ \\
\hline & & & & $\begin{array}{c}\text { Kidney composite } \\
\text { (ESRD, Sustained > } \\
40 \% \text { decrease }\end{array}$ \\
Worsening nephropathy\# & $1.08(0.88-1.32)$ & & & $\begin{array}{c}0.04 \\
\text { in eGFR, or renal death) } \\
1.04(0.89-1.22)\end{array}$ \\
\hline
\end{tabular}

Table 4: Cardiovascular outcome trials of DPP4 inhibitors.

Note: ACS: Acute Coronary Syndrome; CHF: Congestive Heart Failure; CV: Cardiovascular; CVD: Cardiovascular Disease; DPP4: Dipeptidyl Peptidase 4; eGFR: Estimated Glomerular Filtration Rate; ESRD: End-Stage Renal Disease, GLP-1: Glucagon Like Peptide 1; HF: Heart Failure; MACE: Major Adverse Cardiac Event; MI: Myocardial Infraction; UL: Upper Limit.

Data from this table was adapted fro Cephalu, et al. in the January 2020 issue of Diabetes Care.** Age was reported as means in all the trials except EXAMINE, which reported medians; diabetes duration was reported as means in all trials except SAVOR-Timi 53 and EXAMINE, which reported medians. \& Outcome reported as hazard ratio $(95 \% \mathrm{CI})$. \#Worsening nephropathy is defined as doubling of creatinine level, initiation of dialysis, renal transplantation, or creatinine $>6.0 \mathrm{mg} / \mathrm{dl}$ ( $530 \mathrm{mmol} / \mathrm{L})$ in SAVOR -TIMI 53. Worsening nephropathy was a prespecified exploratory adjudicated outcome in SAVOR-TIMI 53. A significant difference in A1C between groups $(\mathrm{p}<0.05)$.

\section{Evidence of SGLT2 Inhibitors in Renal Protection}

This class of anti-diabetic agents was introduced to treat type 2 diabetes mellitus patients in 2012 [11]. They have a physiological mode of action in the kidney, by blocking SGLT2 co-transporter expressed in the proximal convoluted tubule of the nephron and reduces glucose reabsorption leading to increase glucose elimination by urine [12].

There are five recent major trials of three members of the SGLT2i family published, where the renal safety/ renal protection parameters were also evaluated $[13,14]$.
Out of all these trials, EMPA-REG trial was the first clinical trial examining the effects of empagliflozin compared to placebo on cardiovascular morbimortality in patients with type 2 diabetes and at high risk for cardiovascular events in patients with the standard of care $[15,16]$. After EMPA-REG, CANVAS Program, DECLARE -Timi 58, CREDENCE, Dapa- HF, EMPEROR-Reduced and Dapa-CKD trials were published in the last five years [15,17-21].

Out of total seven prospective cardiovascular and renal outcome trials with three commonly used SGLT2 inhibitors, patients who had a moderate renal failure (eGFR $<60 \mathrm{ml} /$ min) ranged from 7.4\% in DECLARE TIMI -58 trial to 59.8\% in CREDENCE trial. Only four of these seven completed trials, Dapa-HF , CREDENCE, EMPEROR-Reduced and Dapa CKD trials have patients on SGLT2i with eGFR upto $30 \mathrm{ml} /$ min and even below ( $<30 \mathrm{ml} / \mathrm{min}$ ) as per baseline inclusion criteria [14,19-21]. In the three prospective cardiovascular outcome trials (EMPA-REG, CANVAS Prog, DECLARE-TIMI 58) majority of patients had low urinary albumin creatinine value (UACR) $<30 \mathrm{mg} / \mathrm{gm}$ ) (Range $59.5 \%$ to $69.1 \%$ ). Only two trials with SGT2i having primary renal endpoints, CREDENCE \& Dapa-CKD had UACR $>300 \mathrm{mg} / \mathrm{g}$ had UACR $>200 \mathrm{mg} / \mathrm{g}$ as inclusion criteria respectively, in terms of albuminuria. In the CREDENCE trial, $99.9 \%$ of patients and in Dapa CKD 97\% of patients were treated with RAAS inhibitors (ARB or ACE inhibitor) (Tables 5, 6 \& 7) [13,22-24]. 
Diabetes \& Obesity International Journal

\begin{tabular}{|c|c|c|c|}
\hline & Empa-Reg & Canvas & Declare \\
\hline Drug & $\begin{array}{c}\text { Empagliflozin } 10 \mathrm{mg}, \\
25 \mathrm{mg} \text { once daily }\end{array}$ & \begin{tabular}{|c|} 
Canagliflozin $100 \mathrm{mg}$, \\
$300 \mathrm{mg}$ once daily
\end{tabular} & Dapagliflozin $10 \mathrm{mg}$ once daily \\
\hline Total of participants & 7,020 & 10,142 & 17,160 \\
\hline $\mathrm{N}(\%)$ with T2D & $7,020(100 \%)$ & $10,142(100 \%)$ & $17,160(100 \%)$ \\
\hline $\mathrm{N}(\%)$ with CVD & $7,020(100 \%)$ & $6,656(66 \%)$ & $6,974(41 \%)$ \\
\hline $\begin{array}{l}\text { eGFR criteria for enrolment } \\
\left(\mathrm{mL} / \mathrm{min} / 1.73 \mathrm{~m}^{2}\right)\end{array}$ & $>30$ & $>30$ & $\mathrm{CrCl}>60 \mathrm{~mL} / \mathrm{min} 45 \%$ had eGFR $60-90$ \\
\hline $\begin{array}{l}\text { Mean eGFR at enrolment } \\
\left(\mathrm{mL} / \mathrm{min} / 1.73 \mathrm{~m}^{2}\right)\end{array}$ & 74 & 76 & 85 \\
\hline $\mathrm{N}(\%)$ with eGFR $<60$ & $1,819(26 \%)$ & $2,039(20 \%)$ & $1,265(7.4 \%)$ \\
\hline ACR & $\begin{array}{c}\text { No criteria. ACR }<30 \\
\mathrm{mg} / \mathrm{g} \text { in } 60 \%, 30-300 \\
\mathrm{mg} / \mathrm{g} \text { in } 30 \%,>300 \\
\mathrm{mg} / \mathrm{g} \text { in } 10 \%\end{array}$ & $\begin{array}{l}\text { No criteria. Median ACR } \\
12.3 \mathrm{mg} / \mathrm{g}\end{array}$ & No criteria \\
\hline Follow-up (median, yrs) & 3.1 & 2.4 & 4.2 \\
\hline Primary outcome & MACE & MACE & MACE \\
\hline CV outcome results & $\begin{array}{c}\text { MACE: (HR } 0.86(0.74, \\
0.99)) ; \text { Hospitalization } \\
\text { for HF (HR 0.65(0.50, } \\
0.85))\end{array}$ & $\begin{array}{c}\text { MACE: (HR } 0.86(0.75, \\
0.97)) ; \text { Hospitalization } \\
\text { for HF (HR 0.67(0.52, } \\
0.87) \text { ) }\end{array}$ & $\begin{array}{c}\text { MACE: (HR } 0.93(0.84,1.03)) \\
\text { Hospitalization for HF }(\text { HR } 0.83(0.73 \text {, } \\
0.95))\end{array}$ \\
\hline Kidney outcome & $\begin{array}{l}\text { Incident of worsening } \\
\text { nephropathy } \\
\text { (progression of } \\
\text { severely increased } \\
\text { albuminuria, doubling } \\
\text { of SCr, initiation of } \\
\text { KRT, or Kidney } \\
\text { Death) and incident } \\
\text { albuminuria } \\
\end{array}$ & $\begin{array}{l}\text { Composite doubling in } \\
\text { SCr, ESKD or death from } \\
\text { kidney causes }\end{array}$ & $\begin{array}{l}>40 \% \text { decrease in eGFR to }<60 \mathrm{ml} / \\
\text { min } / 1.73 \mathrm{~m}^{2}, \\
\text { ESRD, or death from renal cause }\end{array}$ \\
\hline Kidney outcome results & $\begin{array}{c}\text { Incident of worsening } \\
\text { nephropathy; } 12.7 \% \text { vs } \\
18.8 \% \text { in empagliflozin } \\
\text { vs placebo. [HR } \\
0.61(0.53,0.70)] \\
\text { Incident albuminuria: } \\
\text { NS } \\
\end{array}$ & $\begin{array}{c}\text { Composite kidney: } 1.5 \\
\text { vs } 2.8 ; 1000 \text { patient } \\
\text { years in the } \\
\text { canagliflozin vs placebo; } \\
\text { [HR } 0.53(0.33,0.84)]\end{array}$ & $\begin{array}{l}\text { Composite renal-specific outcome [HR } 0.53 \\
\qquad(0.43-0.66)]\end{array}$ \\
\hline
\end{tabular}

Table 5: SGLT2 inhibitor landmark trials (Cardiovascular Outcome Trials).

Note: CrCl: Creatinine Clearance; CV: Cardiovascular; CVD: Cardiovascular Disease; eGFR: Estimated Glomerular Filtration Rate; ESKD: End Stage Kidney Disease; GFR: Glomerular Filtration Rate; HF: Heart Failure; HfrEF: Heart Failure with Reduced Ejection Fraction; HR: Hazard Ratio; KRT: Kidney Replacement Therapy; MACE: Major Adverse Cardiovascular Event; MI: Myocardial Infraction; NS: not significant; SCr: Serum Creatinine; SGLT2: Sodium Glucose Co-Transporter 2; T2D: Type 2 Diabetes Mellitus; uACR: Urinary

Albumin Creatinine Ratio. 


\section{Diabetes \& Obesity International Journal}

\begin{tabular}{|c|c|c|}
\hline & Credence & Dapa- CKD \\
\hline Drug & Canagliflozin 100 mg once daily & Dapagliflozin $10 \mathrm{mg}$ once a day versus placebo \\
\hline Total of participants & 4,401 & 4,304 \\
\hline $\mathrm{N}(\%)$ with T2D & $4,401(100 \%)$ & $2,906(67.5 \%)$ \\
\hline $\mathrm{N}(\%)$ with CVD & $2,220(50 \%)$ & $1,610(37.4 \%)$ \\
\hline $\mathrm{N}(\%)$ on RAASi & $4,397(99.9 \%)$ & $4,174(97 \%)$ \\
\hline $\begin{array}{c}\text { eGFR criteria for enrolment } \\
\left(\mathrm{mL} / \mathrm{min} / 1.73 \mathrm{~m}^{2}\right)\end{array}$ & $30-90 \mathrm{~mL} / \mathrm{min} / 1.73 \mathrm{~m}^{2} \mathrm{ACR} 300-5000 \mathrm{mg} / \mathrm{g}$ & $25-75 \mathrm{~mL} / \mathrm{min} / 1.73 \mathrm{~m} 2 \mathrm{ACR} 200-5000 \mathrm{mg} / \mathrm{g}$ \\
\hline $\begin{array}{l}\text { Mean eGFR at enrolment } \\
\left(\mathrm{mL} / \mathrm{min} / 1.73 \mathrm{~m}^{2}\right)\end{array}$ & 56 & 43.1 \\
\hline $\mathrm{N}(\%)$ with eGFR $<60$ & $2,631(59.8 \%)$ & $3,850(89.4 \%)$ \\
\hline ACR & Median ACR $927 \mathrm{mg} / \mathrm{g}$ & Median ACR $949.5 \mathrm{mg} / \mathrm{g}$ \\
\hline Follow-up (median, yrs) & 2.62 & 2.4 \\
\hline Primary outcome & $\begin{array}{l}\text { Composite Endpoint of Doubling of Serum } \\
\text { Creatinine, End-stage Kidney Disease (ESKD), } \\
\text { and Renal or Cardiovascular (CV) Death }\end{array}$ & $\begin{array}{c}\geq 50 \% \text { sustained decline in eGFR or reaching ESRD or CV } \\
\text { death or renal death }\end{array}$ \\
\hline Kidney outcome results & HR $0.70(0.59,0.82)$ & HR $0.61(0.51-0.72)$ \\
\hline $\mathrm{CV}$ outcome results & $\begin{array}{l}\text { CV death, MI, stroke: }(\text { HR } 0.80(0.67,0.95)) ; \\
\text { Hospitalization for HF }(\text { HR } 0.61(0.47,0.80))\end{array}$ & $\begin{array}{c}\text { Composite of death from cardiovascular causes or } \\
\text { hospitalization for heart failure, (HR } 0.71(0.55,0.92))\end{array}$ \\
\hline All cause mortality & HR $0.83(0.68,1.02)$ & HR $0.69(0.53,0.88)$ \\
\hline
\end{tabular}

Table 6: SGLT2 inhibitor landmark trials (Renal Outcome Trials).

Note: CV: Cardiovascular; CVD: Cardiovascular Disease; eGFR: Estimated Glomerular Filtration Rate; ESKD: End Stage Kidney Disease; GFR: Glomerular Filtration Rate; HF: Heart Failure; HR: Hazard Ratio; RAASi: Renin-Angiotensin-Aldosterone System (RAAS) Inhibitors.

\begin{tabular}{|c|c|c|}
\hline & Dapa-HF & EMPEROR-Reduced \\
\hline Drug & $\begin{array}{c}\text { Dapagliflozin } 10 \text { mg once a day versus } \\
\text { placebo }\end{array}$ & Empagliflozin $10 \mathrm{mg}$ once a day versus placebo \\
\hline Total of participants & 4,474 & 3,730 \\
\hline $\mathrm{N}(\%)$ with T2D & $2,139(45 \%)$ & $1,856(49.8 \%)$ \\
\hline $\begin{array}{l}\text { eGFR criteria for enrolment } \\
\left(\mathrm{mL} / \mathrm{min} / 1.73 \mathrm{~m}^{2}\right)\end{array}$ & $>30$ & $>20$ \\
\hline $\begin{array}{l}\text { Mean eGFR at enrolment (mL/ } \\
\left.\min / 1.73 \mathrm{~m}^{2}\right)\end{array}$ & 66 & 62 \\
\hline $\mathrm{N}(\%)$ with eGFR $<60$ & $1926(41 \%)$ & $1799(48 \%)$ \\
\hline Follow-up (median, yrs) & 1.5 & 1.3 \\
\hline Primary outcome & CV death, HF hospitalization, urgent $\mathrm{HF}$ & CV death, HF hospitalization \\
\hline CV outcome results & Primary: HR (HR $0.74(0.65,0.85))$; & Primary: HR (HR $0.75(0.65,0.86))$; \\
\hline CV Death & HR $0 \cdot 82(0 \cdot 69,0.98)$ & HR $0.92(0 \cdot 75,1 \cdot 12)$ \\
\hline Kidney outcome & $\begin{array}{l}\text { Worsening of Kidney function (identified } \\
\text { as }>50 \text { reductions in eGFR, ESKD or } \\
\text { Kidney death) }\end{array}$ & \begin{tabular}{|c} 
Composite renal endpoint, (defined as time to first \\
occurrence of chronic dialysis; renal transplantation; \\
sustained reduction of $\geq 40 \%$ in estimated GFR; or \\
sustained estimated GFR $<15 \mathrm{~mL} / \mathrm{min} / 1.73 \mathrm{~m} 2$ for \\
patients with baseline estimated GFR $\geq 30 \mathrm{ml} / \mathrm{min} / 1.73 \mathrm{~m}^{2}$ \\
or $<10 \mathrm{~mL} / \mathrm{min} / 1.73 \mathrm{~m}^{2}$ for patients with baseline eGFR \\
$<20 \mathrm{~mL} / \mathrm{min} / 1.73 \mathrm{~m}^{2}$
\end{tabular} \\
\hline Kidney outcome results & HR $0.71(0.44,1.16)$ & HR $0.50(0.32,0.77)$ \\
\hline
\end{tabular}

Table 7: SGLT2 inhibitor landmark trials (Outcome Trials in patient with Heart Failure with Reduced Ejection fraction).

Note: CV: Cardiovascular; CVD: Cardiovascular Disease; eGFR: Estimated Glomerular Filtration Rate; ESKD: End Stage Kidney Disease; GFR: Glomerular Filtration Rate; HF: Heart Failure; HfrEF: Heart Failure with Reduced Ejection Fraction; HR: Hazard Ratio; SGLT2: Sodium Glucose Co-Transporter 2; T2D: Type 2 Diabetes Mellitus; uACR= Urinary albumin creatinine ratio. 


\section{Diabetes \& Obesity International Journal}

Evidence from these seven published event-driven trials has established that SGLT2 inhibitor can provide renal protection, by reducing the risk of dialysis initiation, transplantation, or death due to renal cause. Although the glycaemic benefit of this class of agents was proportional to the glomerular filtration rate, renoprotection was achieved across all levels of baseline renal function categories in these trials $[25,26]$. However, in patients with GFR below $30 \mathrm{ml} / \mathrm{min}$ where currently this class of agents is not yet recommended to be initiated, although the reno-protective benefit has been established by this class in Diabetes (CREDENCE Trial) and both Diabetes and non-diabetes patient (Dapa-CKD Trial) $[13,14,21,27]$. Important cardiovascular outcome trials with SGLT2i have been summarized in Tables 5, 6 \& 7.

\section{Other Safety Parameters}

In the segment of nephrology, specially in the Indian context, infection control is one of the important parameters in Nephrology [2]. From a nephrologist perspective, beyond glycaemic parameters or established cardiovascular or renal safety of anti-diabetic agents, the untoward effects of electrolyte changes, acute kidney injury, etc. also play an important role while choosing an agent. Thus, it is important to understand, safety evidence which is available with these newer classes of antidiabetic agents.

\section{Safety with DPP4 Inhibitors}

DPP4 inhibitors have been available for more than a decade in the Indian market. Nephrologists are well aware of the safety of this class of agents. Few important parameters such as infection, pancreatitis, or pancreatic carcinoma, are points of interest.

Infection: There is no increased risk of infection associated with the majority of trials with different DPP4 inhibitors, other than the initial meta-analysis with sitagliptin. Sitagliptin, in the TECOS trial, however, was not associated with an increased risk of infection $[10,28,29]$.

Pancreatitis \& Pancreatic carcinoma: Across the DPP4i class, both increased levels of pancreatic enzymes or incidence of pancreatitis have been reported [30-32]. Based on these possible safety events, in 2013, MHRA, USFDA, and EMA raised additional concerns of pancreatitis and precancerous cellular changes with DPP4i (Sitagliptin) and GLP1-RA (Exenatide). Following this, all DPP4i class has updated their prescribing information on warning of pancreatitis [33]. With regards to pancreatic carcinoma, the randomized controlled trials did not evidence increased incidence with DPP4 inhibitors $[4,29,34,35]$.

\section{Safety with SGLT2 Inhibitors}

SGLT2 inhibitors are available in the Indian market for the last five years. Still use or adoption of these agents in the segment of nephology is limited. Few important parameters as infection, fractures, amputation, acute metabolic acidosis are points of interest.

Infection: Increased risk of genital mycotic infection has been noted with these drugs, multiple factors like patient immunity, concomitant medication playing an important role. Recently, USFDA has mandated to update the label for Fournier's gangrene, concerning all approved SGLT2i agents in US [36-39]. The infections associated with any SGLT2 inhibitor were mostly mild to moderate, responsive to standard treatments, and usually did not require discontinuation of the medication [40].

Amputation: In the CANVAS program, the incidence of lower limb amputation associated with canagliflozin treatment was significantly higher compared to standard of care ( Hazard Ratio 1.97, 95\% CI 1.41-2.75) [17]. However, later in the CREDENCE trial, there was no increase in the risk of amputation with this drug [19]. Dapagliflozin \& Empagliflozin were not associated with lower limb amputation in the trials $[18,41]$.

Fracture: In the CANVAS program, the incidence of fracture associated with Canagliflozin treatment was significantly higher compared to the standard of care ( $4 \%$ in Canagliflozin vs $2.6 \%$ in placebo). However, later analyses which included 58 peer-reviewed studies including 38,670 patients, did not show an increased risk of fracture with SGLT2 inhibitors [42]. Electrolyte Imbalance: All three SGLT2 inhibitors, Canagliflozin, Dapagliflozin, and Empagliflozin, were associated with a statistically significant increase in serum magnesium levels. However, dapagliflozin only increased serum magnesium levels at the $10 \mathrm{mg}$ dose. Both hypernatremia (Empagliflozin) and hyponatremia (Canagliflozin) have been observed. Serum phosphate levels are slightly increased, while on SGLT2 inhibitors [43-45]. The possible beneficial effect of Empagliflozin in reducing cardiovascular events in the EMPA-Reg trial may be due to increased levels of magnesium and phosphate [15]. However, increase phosphate levels can also lead to detrimental effects, like reduced bone density or increased incidence of fracture, as was seen in the CANVAS program [46]. Between Dapa-CKD and CREDENCE trial, $\mathrm{K}+$ level $>5.5 \mathrm{mmol} / \mathrm{L}$ was an exclusion criteria only in CREDENCE Trial not in Dapa CKD trial. In Dapa CKD trial, 2.7\% patient were on potassium binders $[19,23]$.

Diabetic Keto-Acidosis and AKI: Euglycemic DKA has been reported in the trials in patients with type 2 diabetes on SGLT2 inhibitors [47]. However multiple evidence has found no increased risk of DKA for patients taking SGLT2 inhibitors compared to standard of care $[48,49]$. AKI was noted in patients with history or state of dehydration, in trials or FAERS database analysis [50].

Oncogenic Progression: There is contradictory data with regards to oncogenic progression and SGLT2i. A recent meta- 
analysis found a statistically significant increased risk of bladder cancer, with empagliflozin (OR 3.87 [95\% CI 1.48, 10.09]). Canagliflozin was associated with a statistically significant reduction in the incidence of gastrointestinal cancer vs placebo. Another meta-analysis of 27 clinical trials found no statistically significant increased risk of any type of cancer with SGLT2 inhibitors [51].

\section{DPP4i and SGLT2 i in CKD T2DM- Recommendations}

Based on different important guidelines viz AACE, ADA, and KDIGO, this panel endorses to prioritize these two newer classes of anti-diabetic agents to manage Type 2 Diabetes Mellitus patients associated with CKD as appropriate. This panel has evaluated available evidence of renal safety, cardiovascular safety, baseline cardiovascular risk category, baseline renal function, anticipated complications of chronic kidney disease, type of dialysis, or not on dialysis as well as socioeconomic status, with these newer classes of agents. After evaluation, this panel has come to a consensus suitable for the Indian scenario. The following algorithm has prepared based on the consensus of the panel.

\section{DPP4i and SGLT2i in T2DM and CKD- Algorithm}

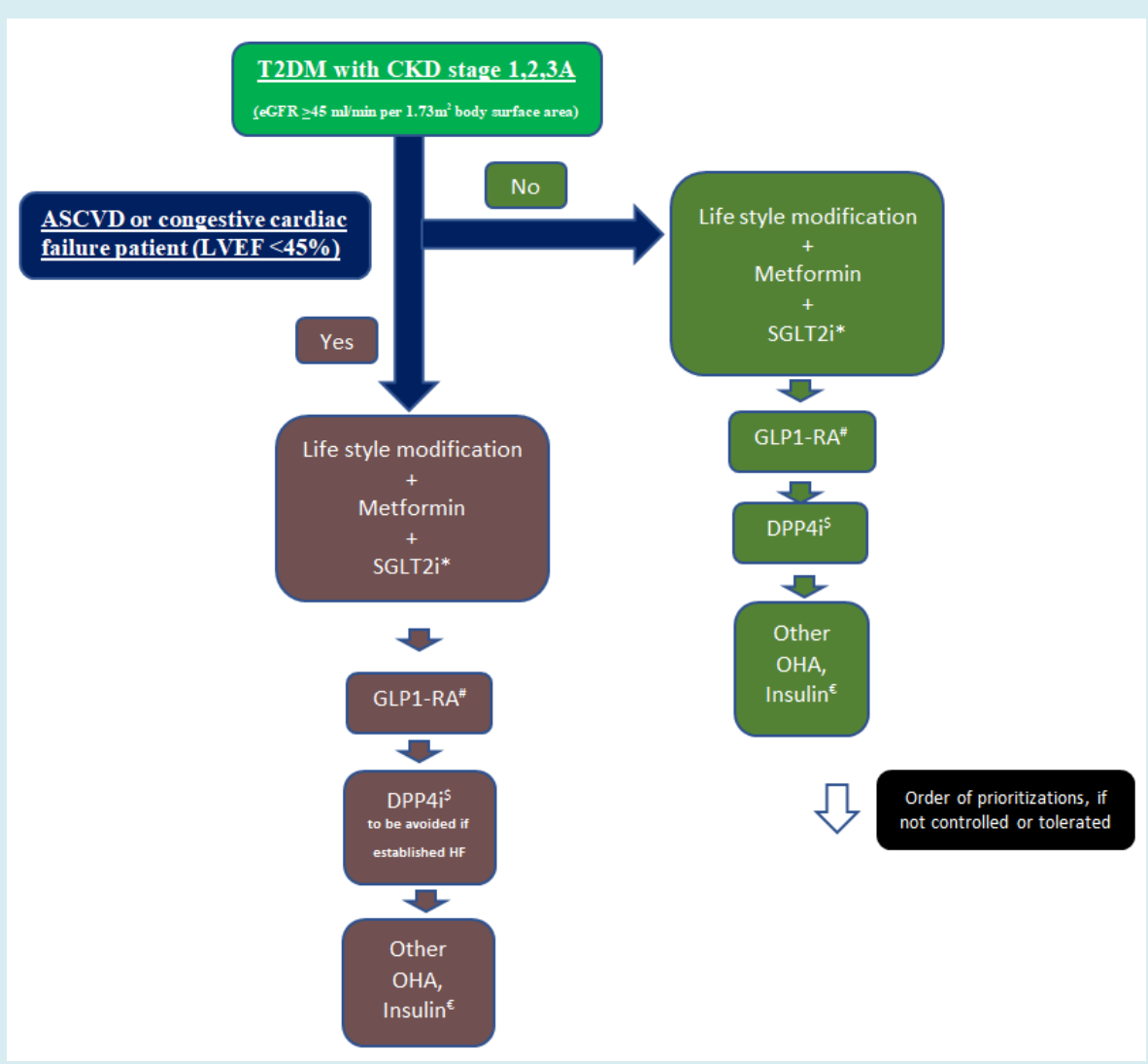

*: SGLT2i with evidence of cardiovascular safety, can be continued if eGFR $<30 \mathrm{ml} / \mathrm{min}$ if tolerated or unless development of uremic symptoms or other complications of CKD. For ASCVD, Empagliflozin and Canagliflozin can be prioritised , for HFrEF $<45 \%$, Dapagliflozin \& Empagliflozin can be prioritised. SGLT2i to be avoided in patient of recurrent history of UTI \& GTI/GMI/GI, dehydration status or catabolic status or kidney transplant recipient.

\#: GLP1-RA (preferable long acting) with established CV safety evidence, can be continued till eGFR $>30 \mathrm{ml} / \mathrm{min}$. For GLP1-RA- cost and injectable/parenteral route would make it less preferable over DPP4i.

\$: DPP4i with established CV safety evidence can be considered.

$€:$ as applicable.

Note :

- $\quad$ SGLT2i \& GLP1-RA or SGLT2i \& DPP4i- these combination can be prescribed together.

- $\quad$ Patient should not be prescribed combination of GLP1-RA and DPP4i. 


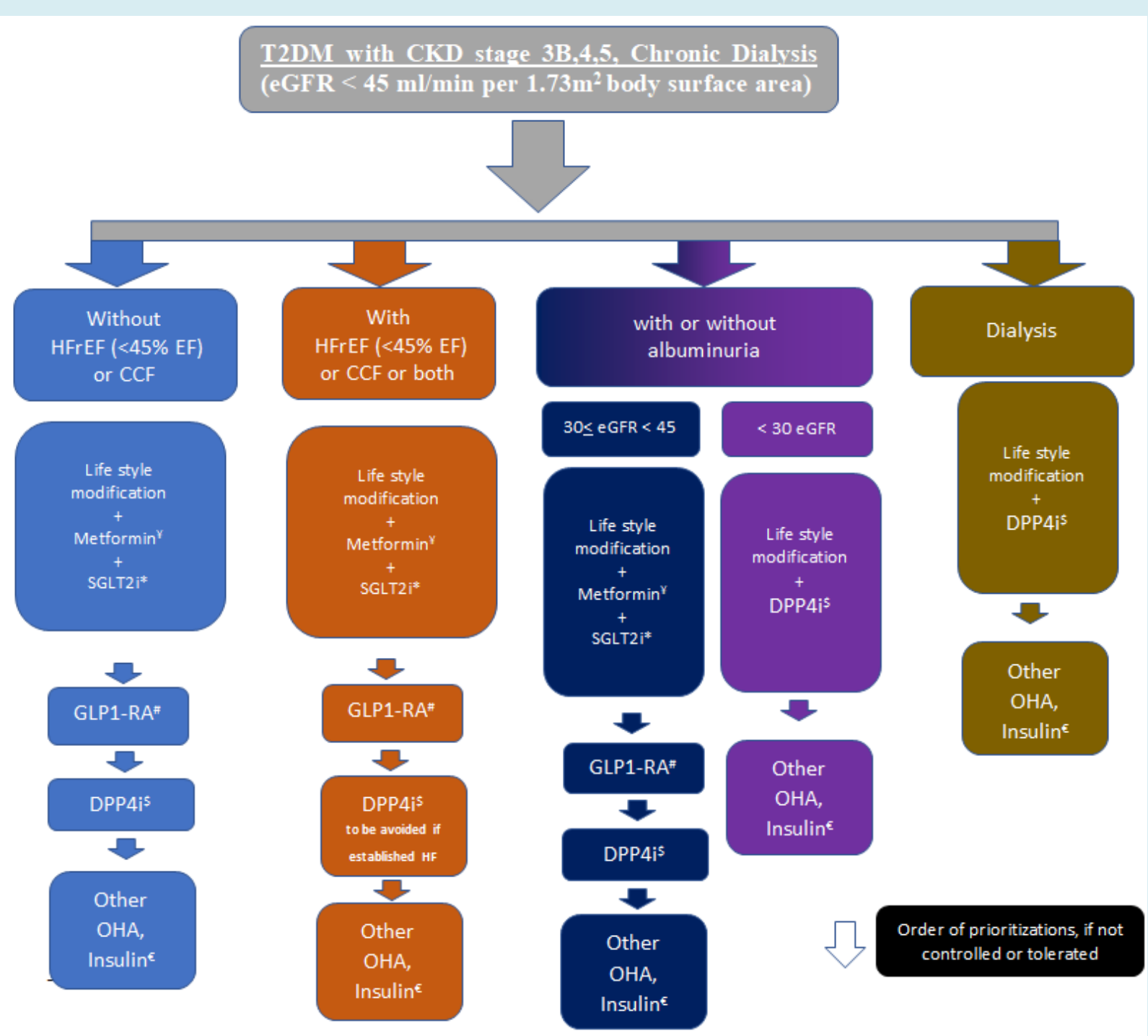

$¥:$ Metformin can be continued till eGFR $>30 \mathrm{ml} / \mathrm{min}$, should be discontinued eGFR $<30 \mathrm{ml} / \mathrm{min}$.

*: SGLT2i with evidence of cardiovascular safety, can be continued eGFR $<30 \mathrm{ml} / \mathrm{min}$ if tolerated unless development of uremic symptoms or other complications of CKD. For ASCVD, Empagliflozin and Canagliflozin can be prioritised, for HFrEF $<45 \%$, Dapagliflozin \& Empagliflozin can be prioritised. For patient with albuminuria, Canagliflozin \& Dapagliflozin can be prioritized, the rest of SGLT2i can also be considered based on available evidence. Contraindicated in dialysis patient. SGLT2i to be avoided in patient of recurrent history of UTI \& GTI/GMI/GI, dehydration status or catabolic status or kidney transplant recipient.

\#: GLP1-RA (preferable long acting) with established CV safety evidence, can be continued till eGFR $>30 \mathrm{ml} / \mathrm{min}$. Discontinue if eGFR $<30 \mathrm{ml} / \mathrm{min}$. For GLP1-RA cost and injectable/parenteral route would make it less preferable over DPP4i.

\$: DPP4i with established CV safety evidence can be considered. DPP4i with renal safety evidence (Saxagliptin or Linagliptin) can be prioritized. Rest of DPP4 inhibitors like Sitagliptin, Alogliptin, Vildagliptin and others can also be considered. For Hemodialysis patient, Sitagliptin, Saxagliptin, Alogliptin can be considered. Evidences with Linagliptin and Vildagliptin in Dialysis are limited. For peritoneal dialysis patient, evidence with DPP4 inhibitor is currently limited. Can be used with caution if use is mandatory.

$€$ : as applicable.

Note :

- $\quad$ SGLT2i \& GLP1-RA or SGLT2i \& DPP4i- these combination can be prescribed together.

- Patient should not be prescribed combination of GLP1-RA and DPP4i. 


\section{Conclusion}

In T2DM patients with comorbidities, both SGLT2 inhibitors and GLP1-RA are safe, well-tolerated, and have been extensively studied for efficacy and cardio-renal safety. T2DM patients in all stages (excluding Dialysis at baseline) of CKD have been included in these studies. Both these classes of agents have a low propensity for hypoglycemia.

These two classes of agents can be endorsed in diabetic CKD patients, based on favorable profiles, and their ability to prevent the progression of CKD.

DPP4 inhibitors have established renal safety, have demonstrated cardiovascular neutrality and most of them have an anti-proteinuric effect. Hence, this group can be considered as OHA in diabetic CKD patients, as an alternative to GLP1-RA in developing countries like India given the latter's cost and the need for the parenteral route.

We hope this simple and updated algorithm, updated with both SGLT2i \& DPP4i based on renal safety evidence, will help Indian nephrologists to treat diabetes CKD patients more efficiently.

\section{Conflict of Interest}

Kaushik Mandal is an employee of AstraZeneca Pharma India Ltd. The rest of the authors, do not have any conflict of interest.

\section{Funding}

AstraZeneca Pharma India Limited has assisted to prepare the document and only funded towards publication.

\section{References}

1. Abraham G, Varughese $\mathrm{S}$, Thandavan $\mathrm{T}$, Iyengar $\mathrm{A}$, Fernando E, et al. (2016) Chronic kidney disease hotspots in developing countries in South Asia. Clinical kidney journal 9(1): 135-141.

2. Varughese S, Abraham G (2018) Chronic Kidney Disease in India. A Clarion Call for Change. Clin J Am Soc Nephrol 13(5): 802-804.

3. Jafar TH, Ramakrishnan C, John 0, Tewari A, Cobb B, et al. (2020) Access to CKD Care in Rural Communities of India: a qualitative study exploring the barriers and potential facilitators. BMC Nephrology 21(1): 26.

4. Scirica BM, Bhatt DL, Braunwald E, Steg PG, Davidson J, et al. (2013) Saxagliptin and Cardiovascular Outcomes in Patients with Type 2 Diabetes Mellitus. N Engl J Med 369(14): 1317-1326.

\section{Diabetes \& Obesity International Journal}

5. Udell JA, Bhatt DL, Braunwald E, Cavender MA, Mosenzon 0, et al. (2015) Saxagliptin and Cardiovascular Outcomes in Patients With Type 2 Diabetes and Moderate or Severe Renal Impairment: Observations From the SAVOR-TIMI 53 Trial. Diabetes Care 38(4): 696-705.

6. Mosenzon O, Leibowitz G, Bhatt DL, Cahn A, Hirshberg B, et al. (2017) Effect of Saxagliptin on Renal Outcomes in the SAVOR-TIMI 53 Trial. Diabetes Care 40(1): 69-76.

7. Scirica BM, Braunwald E, Raz I, Cavender MA, Morrow DA, et al. (2014) Heart failure, saxagliptin, and diabetes mellitus: observations from the SAVOR-TIMI 53 randomized trial. Circulation 130(18): 1579-1588.

8. Groop PH, Cooper M, Perkovic V, Hocher B, Kanasaki K, et al. (2017) Linagliptin and its effects on hyperglycaemia and albuminuria in patients with type 2 diabetes and renal dysfunction: The randomized MARLINA-T2D trial. Diabetes, obesity \& metabolism 19(11): 1610-1619.

9. Rosenstock J, Perkovic V, Alexander JH, Cooper ME, Marx N, et al. (2018) Rationale, design, and baseline characteristics of the CArdiovascular safety and Renal Microvascular outcomE study with LINAgliptin (CARMELINA((R))): a randomized, double-blind, placebo-controlled clinical trial in patients with type 2 diabetes and high cardio-renal risk. Cardiovascular diabetology 17(1): 39 .

10. Richter B, Bandeira-Echtler E, Bergerhoff K, Lerch CL (2008) Dipeptidyl peptidase-4 (DPP-4) inhibitors for type 2 diabetes mellitus. The Cochrane database of systematic reviews 2: CD006739.

11. (2013) Center for Drug Evaluation and Research. Application Number: 2022930rig1s000, USFDA.

12. Anderson SL (2014) Dapagliflozin efficacy and safety: a perspective review. Ther Adv Drug Saf 5(6): 242-254.

13. Neuen BL, Young T, Heerspink HJL, Neal B, Perkovic V, et al. (2019) SGLT2 inhibitors for the prevention of kidney failure in patients with type 2 diabetes: a systematic review and meta-analysis. The lancet Diabetes \& endocrinology 7(11): 845-854.

14. McMurray JJV, Solomon SD, Inzucchi SE, Køber L, Kosiborod MN, et al. (2019) Dapagliflozin in Patients with Heart Failure and Reduced Ejection Fraction. N Engl J Med 381(21): 1995-2008.

15. Zinman B, Wanner C, Lachin JM, Fitchett D, Bluhmki E, et al. (2015) Empagliflozin, Cardiovascular Outcomes, and Mortality in Type 2 Diabetes. The New England journal of medicine 373(22): 2117-2128. 


\section{Diabetes \& Obesity International Journal}

16. Barnett AH, Mithal A, Manassie J, Jones R, Rattunde H, et al. (2014) Efficacy and safety of empagliflozin added to existing antidiabetes treatment in patients with type 2 diabetes and chronic kidney disease: a randomised, double-blind, placebo-controlled trial. The lancet Diabetes \& endocrinology 2(5): 369-384.

17. Neal B, Perkovic V, Mahaffey KW, de Zeeuw D, Fulcher G, et al. (2017) Canagliflozin and Cardiovascular and Renal Events in Type 2 Diabetes 377(7): 644-657.

18. Wiviott SD, Raz I, Bonaca MP, Mosenzon O, Kato ET, et al. (2018) Dapagliflozin and Cardiovascular Outcomes in Type 2 Diabetes. N Engl J Med 380(4): 347-357.

19. Perkovic V, Jardine MJ, Neal B, Bompoint S, Heerspink HJL, et al. (2019) Canagliflozin and Renal Outcomes in Type 2 Diabetes and Nephropathy. N Engl J Med 380(24): 2295-2306.

20. Packer M, Anker SD, Butler J, Filippatos G, Pocock SJ, et al. (2020) Cardiovascular and Renal Outcomes with Empagliflozin in Heart Failure. N Engl J Med 383(15): 1413-1424.

21. Heerspink HJL, Stefánsson BV, Correa-Rotter R, Chertow GM, Greene T, et al. (2020) Dapagliflozin in Patients with Chronic Kidney Disease. N Engl J Med 383:1436-1446.

22. Lucas Franki (2020) Diabetes Drug Slows Kidney Disease DAPA-CKD Trial Stopped Early. Medscape.

23. Wheeler DC, Stefansson BV, Batiushin M, Bilchenko O, Cherney DZI, et al. (2020) The dapagliflozin and prevention of adverse outcomes in chronic kidney disease (DAPA-CKD) trial: baseline characteristics. Nephrology Dialysis Transplantation 35(10): 17001711.

24. Jardine MJ, Mahaffey KW, Neal B, Agarwal R, Bakris GL, et al. (2017) The Canagliflozin and Renal Endpoints in Diabetes with Established Nephropathy Clinical Evaluation (CREDENCE) Study Rationale, Design, and Baseline Characteristics. Am J Nephrol 46(6): 462-472.

25. Heerspink HJ, Perkins BA, Fitchett DH, Husain M, Cherney DZ (2016) Sodium Glucose Cotransporter Inhibitors in the Treatment of Diabetes Mellitus: Cardiovascular and Kidney Effects, Potential Mechanisms, and Clinical Applications. Circulation 134(10): 752-772.

26. Heerspink HJL, Kosiborod M, Inzucchi SE, Cherney DZI (2018) Renoprotective effects of sodium-glucose cotransporter-2 inhibitors. Kidney international 94(1): 26-39.

27. Kidney Disease: Improving Global Outcomes (KDIGO)
Diabetes Work Group (2020) KDIGO 2020 Clinical Practice Guideline for Diabetes Management in Chronic Kidney Disease. Kidney International 98(S4): S1-S115.

28. Amori RE, Lau J, Pittas AG (2007) Efficacy and safety of incretin therapy in type 2 diabetes: systematic review and meta-analysis. Jama 298(2): 194-206.

29. Green JB, Bethel MA, Armstrong PW, Buse JB, Engel SS, et al. (2015) Effect of Sitagliptin on Cardiovascular Outcomes in Type 2 Diabetes. N Engl J Med 373(3): 232242.

30. Merck Sharp, Dohme Corp (2012) Sitagliptin prescribing information.

31. Girgis CM, Champion BL (2011) Vildagliptin-induced acute pancreatitis. Endocrine practice: official journal of the American College of Endocrinology and the American Association of Clinical Endocrinologists 17(3): e48-e50.

32. Ligueros-Saylan M, Foley JE, Schweizer A, Couturier A, Kothny W (2010) An assessment of adverse effects of vildagliptin versus comparators on the liver, the pancreas, the immune system, the skin and in patients with impaired renal function from a large pooled database of Phase II and III clinical trials. Diabetes, obesity \& metabolism 12(6): 495-509.

33. Butler AE, Campbell-Thompson M, Gurlo T, Dawson DW, Atkinson M, et al. (2013) Marked expansion of exocrine and endocrine pancreas with incretin therapy in humans with increased exocrine pancreas dysplasia and the potential for glucagon-producing neuroendocrine tumors. Diabetes 62(7): 2595-2604.

34. Rosenstock J, Perkovic V, Johansen OE, Cooper ME, Kahn SE, et al. (2019) Effect of Linagliptin vs Placebo on Major Cardiovascular Events in Adults With Type 2 Diabetes and High Cardiovascular and Renal Risk: The CARMELINA Randomized Clinical Trial. Jama 321(1): 69-79.

35. White WB, Cannon CP, Heller SR, Nissen SE, Bergenstal RM, et al. (2013) Alogliptin after Acute Coronary Syndrome in Patients with Type 2 Diabetes. N Engl J Med 369(14): 1327-1335.

36. Dryden M, Baguneid M, Eckmann C, Corman S, Stephens J, et al. (2015) Pathophysiology and burden of infection in patients with diabetes mellitus and peripheral vascular disease: focus on skin and soft-tissue infections. Clin Microbiol Infect 21(S2): S27-S32.

37. Shah BR, Hux JE (2003) Quantifying the Risk of Infectious Diseases for People With Diabetes. Diabetes Care 26(2): 
510-513.

38. Benfield T, Jensen JS, Nordestgaard BG (2007) Influence of diabetes and hyperglycaemia on infectious disease hospitalisation and outcome. Diabetologia 50(3): 549554.

39. Scheen AJ (2019) An update on the safety of SGLT2 inhibitors. Expert opinion on drug safety 18(4): 295311.

40. Bode B, Stenlof K, Harris S, Sullivan D, Fung A, et al. (2015) Long-term efficacy and safety of canagliflozin over 104 weeks in patients aged 55-80 years with type 2 diabetes. Diabetes, obesity \& metabolism 17(3): 294303.

41. Inzucchi SE, Iliev H, Pfarr E, Zinman B (2018) Empagliflozin and Assessment of Lower-Limb Amputations in the EMPA-REG OUTCOME Trial. Diabetes Care 41(1): e4-e5.

42. Ruanpeng D, Ungprasert P, Sangtian J, Harindhanavudhi $\mathrm{T}$ (2017) Sodium-glucose cotransporter 2 (SGLT2) inhibitors and fracture risk in patients with type 2 diabetes mellitus: A meta-analysis. Diabetes Metab Res Rev 33(6).

43. Tang H, Zhang X, Zhang J, Li Y, Del Gobbo LC, et al. (2016) Elevated serum magnesium associated with SGLT2 inhibitor use in type 2 diabetes patients: a meta-analysis of randomised controlled trials. Diabetologia 59(12): 2546-2551.

44. Forst T, Guthrie R, Goldenberg R, Yee J, Vijapurkar U, et al. (2014) Efficacy and safety of canagliflozin over 52 weeks in patients with type 2 diabetes on background metformin and pioglitazone. Diabetes Obes Metab 16(5): 467-477.

\section{Diabetes \& Obesity International Journal}

45. Weir MR, Kline I, Xie J, Edwards R, Usiskin K (2014) Effect of canagliflozin on serum electrolytes in patients with type 2 diabetes in relation to estimated glomerular filtration rate (eGFR). Curr Med Res Opin 30(9): 17591768.

46. Taylor SI, Blau JE, Rother KI (2015) Possible adverse effects of SGLT2 inhibitors on bone. The lancet Diabetes \& endocrinology 3(1): 8-10.

47. Pfützner A, Klonoff D, Heinemann L, Ejskjaer N, Pickup J (2017) Euglycemic ketosis in patients with type 2 diabetes on SGLT2-inhibitor therapy-an emerging problem and solutions offered by diabetes technology. Endocrine 56(1): 212-216.

48. Donnan JR, Grandy CA, Chibrikov E, Marra CA, AubreyBassler K, et al. (2019) Comparative safety of the sodium glucose co-transporter 2 (SGLT2) inhibitors: a systematic review and meta-analysis. BMJ open 9(1): e022577.

49. Saad M, Mahmoud AN, Elgendy IY, Abuzaid A, Barakat AF, et al. (2017) Cardiovascular outcomes with sodiumglucose cotransporter-2 inhibitors in patients with type II diabetes mellitus: A meta-analysis of placebocontrolled randomized trials. International journal of cardiology 228: 352-358.

50. Fralick M, Schneeweiss S, Patorno E (2017) Risk of Diabetic Ketoacidosis after Initiation of an SGLT2 Inhibitor. The New England journal of medicine 376(23): 2300-2302.

51. Dicembrini I, Nreu B, Mannucci E, Monami M (2019) Sodium-glucose co-transporter-2 (SGLT-2) inhibitors and cancer: A meta-analysis of randomized controlled trials. Diabetes, obesity \& metabolism 21(8): 1871-1877. 